\title{
VON WILLEBRAND DISEASE-TYPE2B
}

Sushma Belurkar, Chethan Manohar, Annamma Kurien

1. Associate Professor, Department of Pathology, Kasturba Medical College, Manipal.

2. Professor, Department of Pathology, Kasturba Medical College, Manipal.

3. Professor, Department of Pathology, MelakaManipal Medical College,Manipal.

\section{CORRESPONDING AUTHOR}

Dr. Sushma Belurkar,

Department of Pathology,

Basic Sciences Building,

Kasturba Medical College,Manipal

E-mail: sushbelur@gmail.com

Ph: 00919448888415

ABSTRACT: Type 2B Von Willebrand disease (vWD) is a rare type of vWD accounting for less than $20 \%$ of the cases of vWD. The characteristic feature of this condition is increased affinity of $\mathrm{vWF}$ for the glycoprotein Ib-IX-V complex on platelets resulting in clearing of the high molecular weight vWF multimers and platelets from the circulation. So the patient will present with low levels of vWF as well as thrombocytopenia. This functional abnormality can be demonstrated by hyperaggregation seen with low dose ristocetin on platelet aggregation. Here we present a case of type $2 \mathrm{~B} v W D$ in a seven year old male child who presented with skin bleeds and prolonged bleeding following tooth fall. The case is discussed because of its rarity and to emphasize the careful laboratory evaluation required to avoid misdiagnosis in such cases.

KEYWORDS: von Willebrand factor, glycoproteinIb, ristocetin

INTRODUCTION: Von Willebrand disease(vWD) is the most common human bleeding disorder. Most of the patients have simple quantitative deficiency of von Willebrand factor (vWF) but around $20 \%$ can have qualitative defects in the vWF and are referred to as vWD type 2.Type 2 is further classified into2A,B, $\mathrm{M}$ and $\mathrm{N}$ types.Type2B vWD is a rare type of $v W D$ that is characterized by enhanced affinity of $\mathrm{vWF}$ to aspecific platelet receptor, glycoprotein Ib-IX complex. The characteristic functional abnormality in this variant is demonstrated by ristocetin induced platelet aggregation (RIPA).In type $2 \mathrm{~B} v W D$ very low doses of ristocetin, which have no effect on normal platelet rich plasma result in full aggregation.

CASE REPORT: A 7 year old male child presented with maculopapular rash on the body with bleeding tendency. Patient also had history of prolonged bleeding following tooth fall. There was no history of bleeding from any other sites. There was no positive family or sibling history. Laboratory investigations revealed a prolonged bleeding time( $>15$ minutes) and mildly prolonged APTT(40.4 secs).Platelets were mildly reduced in number $(93000 / \mathrm{cmm})$.RIPA showed $>50 \%$ aggregation with low dose ristocetin $(0.5 \mathrm{mg} / \mathrm{ml})$ and $\mathrm{VWF}$ assay was also mildly reduced (38\%).Hence a diagnosis of type IIbV WD was considered. Hyperaggregation with low dose ristocetin was not corrected by adding normal platelets but was corrected by adding normal plasma, hence platelet type of VWD was ruled out.(Figures 1-4)

DISCUSSION: Type 2B is a qualitative type of vWD. A characteristic gain-of-function phenotype, subtype $2 \mathrm{~B}$, may be the result of single amino acid substitutions within the A1 domain of vWF. 
These changes result in an increased interaction between $\mathrm{VWF}$ and the glycoprotein Ib-IX-V complex in platelets and an acceleration in binding and clearance of vWF and platelets; this interaction can be exacerbated by an increased production of vWF that can be produced with stressful clinical situations. ${ }^{1}$ The gain of function in the vWF A1 domain creates 3 conditions that explain increased tendency for bleeding2:

$>$ The large, hemostatically most effective vWF multimers are decreased in plasma

$>$ Platelet count may be reduced; and

$>$ Part of the GPIb $\alpha$ receptors may be occupied by soluble vWF and unable to interact with vWF immobilized on the tissues exposed at the sites of injury.

Type 2B vWD is generally inherited as an autosomal dominant condition but apparently recessive inheritance has also been reported ${ }^{3}$.The disease is caused by missense mutations occurring in the region of VWF that binds to glycoprotein Ib-IX of the platelets and these mutations can be detected by PCR amplification of cDNA sequences. In our case, the parents did not have any history of bleeding tendency and were never screened for the same.

Patients with type 2B vWD present with mild to moderate mucocutaneous bleeding. Bleeding symptoms may be exaggerated if there is significant thrombocytopenia. Symptoms may worsen with severe infections, pregnancy, surgery and Desmopressin treatment ${ }^{4}$.

The basic laboratory investigations in type $2 \mathrm{~B} v \mathrm{vD}$ reveal a prolonged bleeding, normal or reduced platelet count ,normal or elevated APTT ${ }^{5}$.Thrombocytopenia is usually mild but cases with severe thrombocytopenia have also been reported ${ }^{1}$.Thrombocytopenia may be present due to enhanced ability of vWFto bind platelet receptor GPIbA, causing removal of the platelet/vWF complex. But Nurden et al have shown in their study that severe thrombocytopenia can be seen in type $2 \mathrm{~B}$ vWD due to impaired megakaryopoesis as evidenced both by changes in proteins involved in platelet production and electron microscopy ${ }^{2}$.

The characteristic functional abnormality in this variant is demonstrated by the ristocetin- induced platelet aggregation (RIPA) assay. The antibiotic ristocetin causes dosedependent platelet aggregation when added to platelet-rich plasma, and this aggregation requires the interaction of plasma vWF with platelet GPIb-IX. Most variants of vWD exhibit decreased or absent RIPA, manifested by a reduced extent of aggregation or a requirement for increased concentrations of ristocetin, because of decreased vWF concentration or function. In vWD type IIB, however, very low concentrations of ristocetin, which have no effect on normal platelet rich plasma, result in full platelet aggregation ${ }^{2}$.

Other laboratory findings which aid in diagnosis of vWD type $2 \mathrm{~B}$ are relative decrease in plasma high molecular weight vWFmultimers ${ }^{6}$, disproportionately low ristocetin cofactor activity compared to vWFlevels ${ }^{7}$ and mildly reduced or normal vWF assay. But the gold standard test to confirm the diagnosis of vWD type $2 \mathrm{~B}$ is by molecular analysis of the vWF gene exon 28 sequence.

The challenge lies in distinguishing type $2 \mathrm{~B}$ vWD from the platelet type vWD (PT-vWD) because these two conditions share many clinical and laboratory features. In PT-vWD the defect lies in the GP IbA gene coding for the platelet glycoprotein IB and not in vWF.A simple laboratory method that can be used to distinguish the two is by doing RIPA mixing assays. The 
hyperaggregation with low dose ristocetin in type $2 \mathrm{~B} v \mathrm{vD}$ will get corrected by adding normal plasma to patient's platelet rich plasma(PRP) whereas in PT-vWD it will get corrected by adding normal platelets to patient's platelet poor plasma (PPP) ${ }^{8}$.RIPA mixing assay was done and the correction was seen by adding normal plasma to patient's PRP, hence PT-vWD was ruled out but more confirmatory tests like flow cytometry and genetic studies were not done as these facilities were not available in our institution.

Definitive diagnosis of vWD type 2B is critical for treatment decisions. Although Desmopressin, a vasopressin analogue that raises plasma vWF is a common therapy for vWD, it is contraindicated in type $2 \mathrm{~B} v \mathrm{vW}$ as it can further exaggerate the thrombocytopenia and bleeding potentials ${ }^{1}$. The preferred therapy for type $2 \mathrm{~B} \mathrm{vWD}$ is $\mathrm{vWF} /$ factor VIII plasma concentrates and if there is significant thrombocytopenia, platelet concentrates should also be used.

CONCLUSION: Type 2B vWD is a rare type of vWD, which is characterised by selective loss of vWF multimers in plasma due to their abnormally increased reactivity with platelets. The two important features which differentiates type 2B vWD from other subtypes are thrombocytopenia and hyperaggregation with low dose ristocetin. Definitive diagnosis of type $2 \mathrm{~B} \mathrm{vWD}$ is essential for correct treatment decisions.

\section{REFERENCES:}

1. Hapner,David L.,Tsen,LawrenD.Severethrombocytopenia,Type2B von Willebrand disease and pregnancy.Anaesthesiology 2004; 101(6) :1465-67.

2. FedericiAB.Impairedmegakaryocytopoesis in type 2B von Willebranddisease.Blood 2006;108:2498-99.

3. Randi AM,Robinowitz I, MancusoD.Molecular basis of von Willebravd disease type 2B,candidate mutations cluster in one disulphide loop between proposed platelet GP Ib binding sequences.JClin Invest 1991;87:1220-26.

4. Lion E, Smock K, MillerC.vonWillebrand disease type 2 (VWF) sequencing ,selected exons or platelet type von Willebrand disease(GP1BA),4mutations:ARUP laboratory(National reference laboratory); November 2011.

5. RileyRS:vonWillebranddisease.April 2005.

6. Cooney KA, GinsburgD.Comparative of type 2B von Willebrand disease mutation analysis:implications for the mechanism of platelet binding to platelet.Blood 1996;87:2322-28.

7. Cooney KA, Lyons SE, GinsburgD.Functional analysis of type 2B von Willebrand disease missense mutation:Increased binding of von Willebrand factor multimers to platelets.ProcNaclAcadSci USA 1992;89:2869-72.

8. Othman M.Platelet type von Willebranddisease:a rare often misdiagnosed and underdiagnosed bleeding disorder.SeminThrombHemost 2011;37(5): 464-69

Fig 1 : Platelet aggregation with high dose ristocetin $(1.5 \mathrm{mg} / \mathrm{ml})$ for control $(92 \%)$ and patient $(59 \%)$ (High dose ristocetin platelet aggregation is mildly reduced for the patient) 


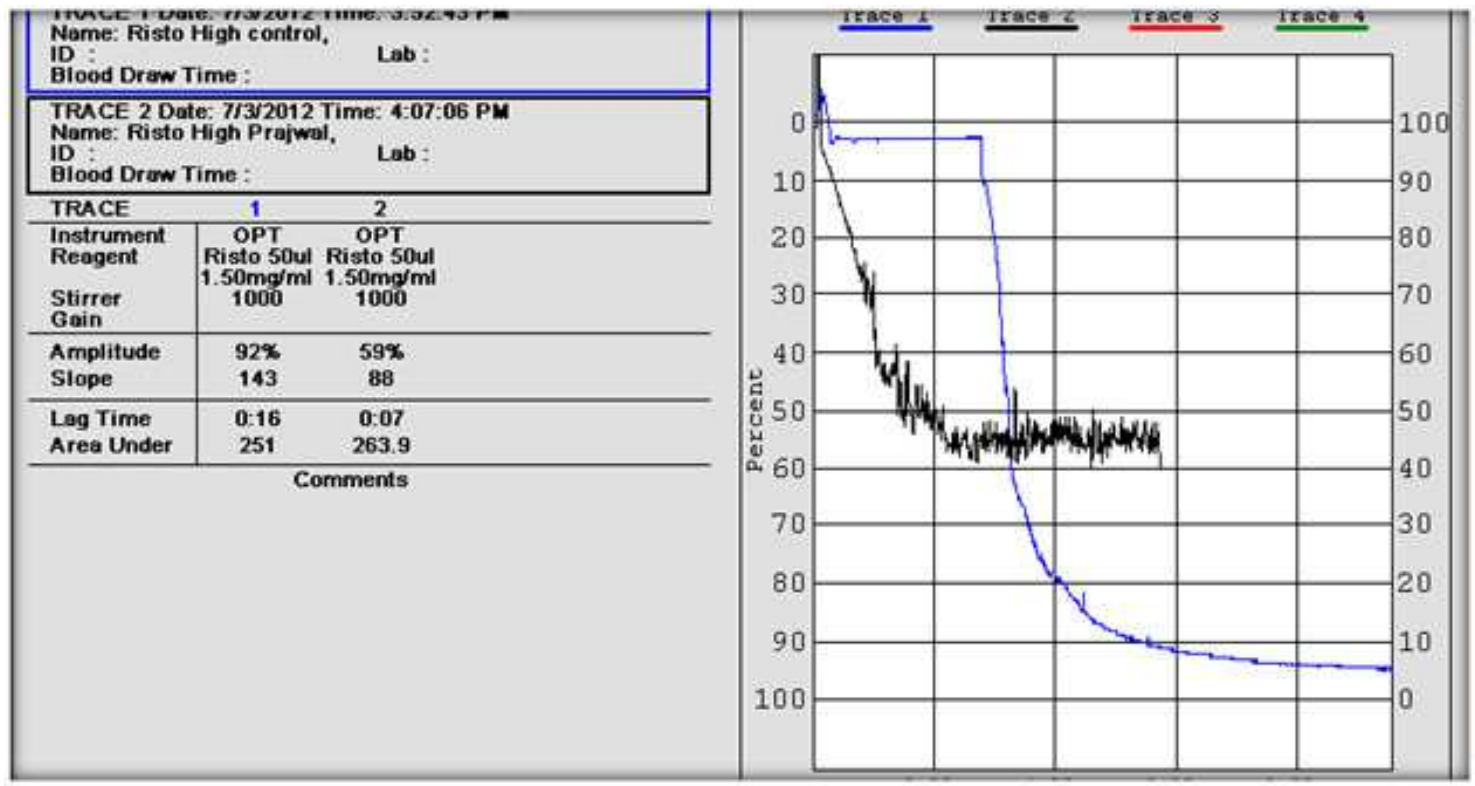

Fig 2: Platelet aggregation with low dose ristocetin $(0.5 \mathrm{mg} / \mathrm{ml})$ for control(3\%) and patient $(53 \%)$ (Low dose ristocetin platelet aggregation is increased for the patient)

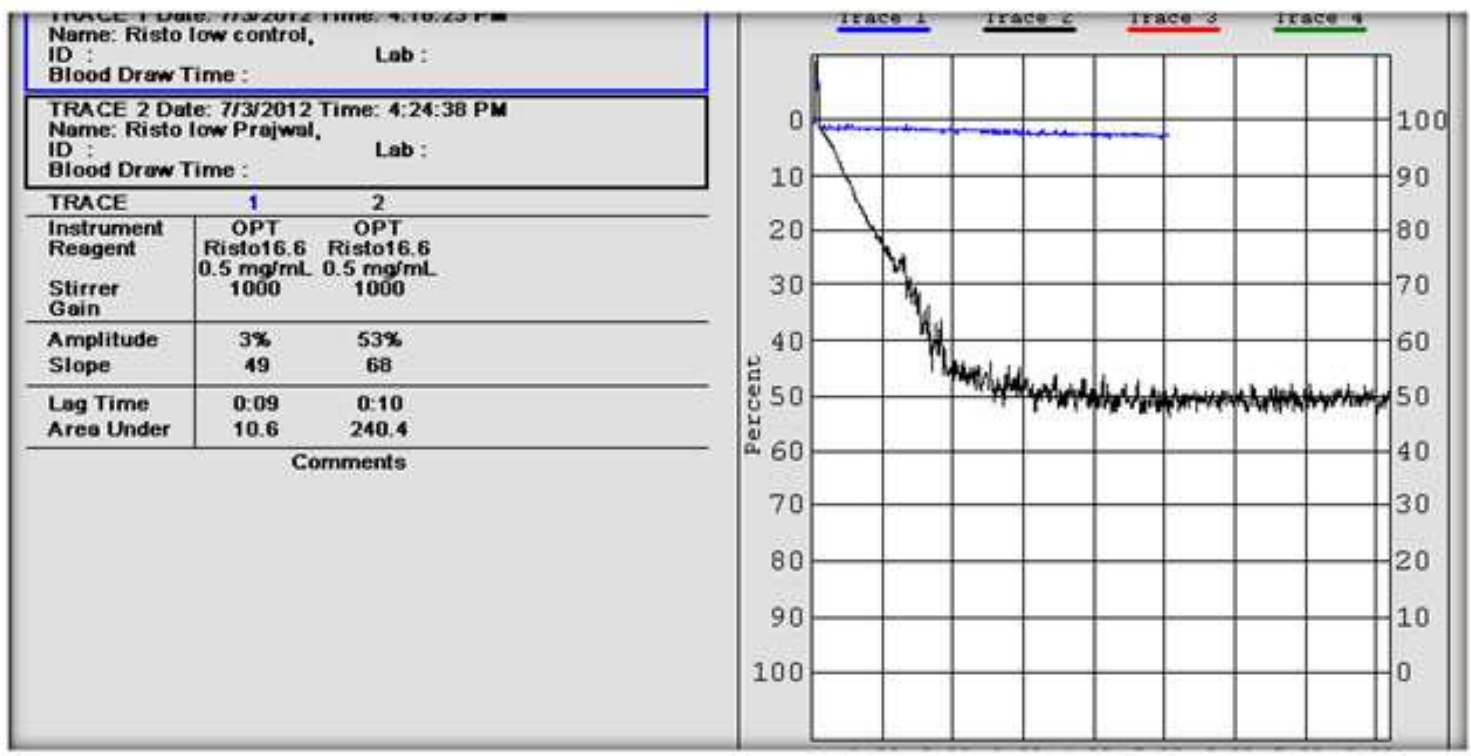


Fig 3: Low dose ristocetin platelet aggregation after mixing normal platelets to patient's $\mathrm{PPP}($ Hyperaggregation with low dose ristcetin is not correctecd)

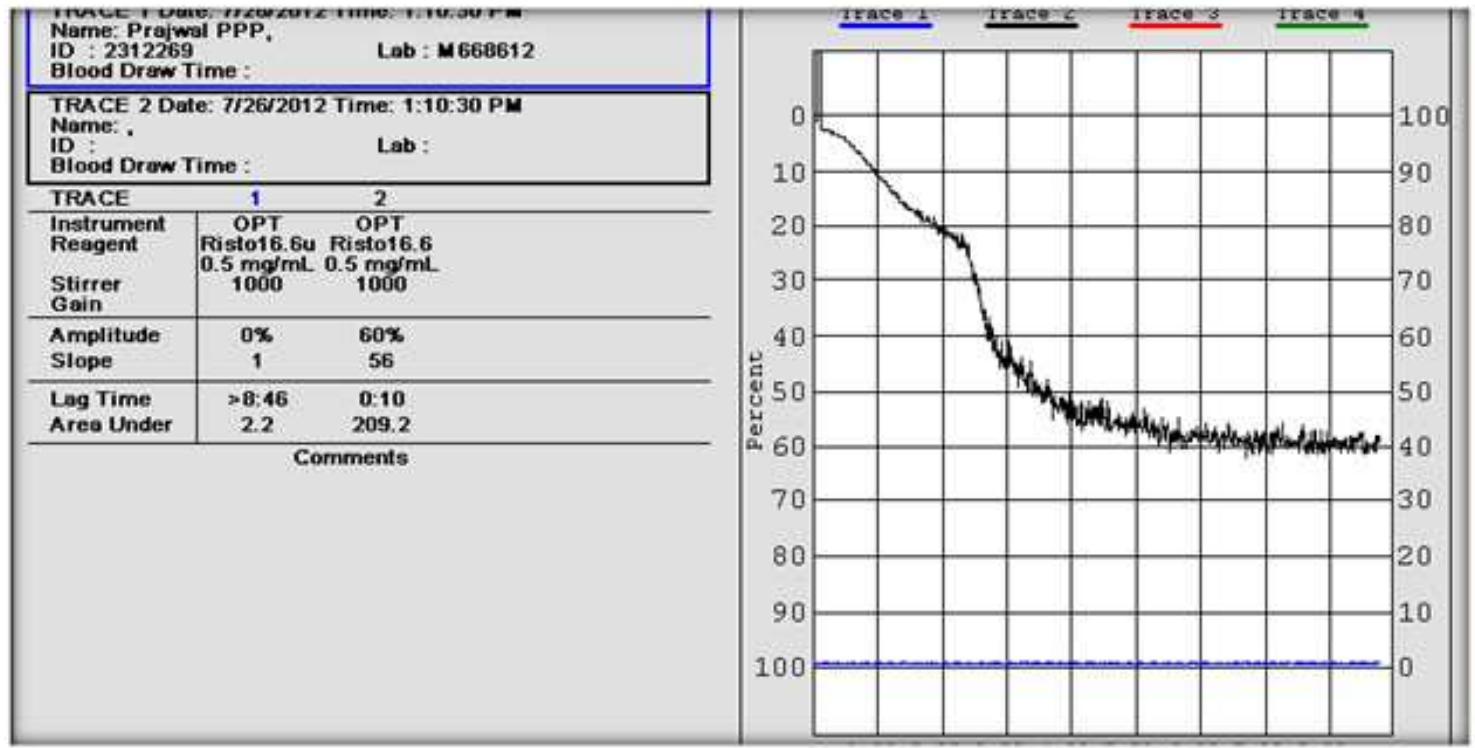

Fig 4:Low dose ristocetin platelet aggregation after mixing normal plasma to patient's PRP (Hyperaggregation with low dose ristocetin is corrected)

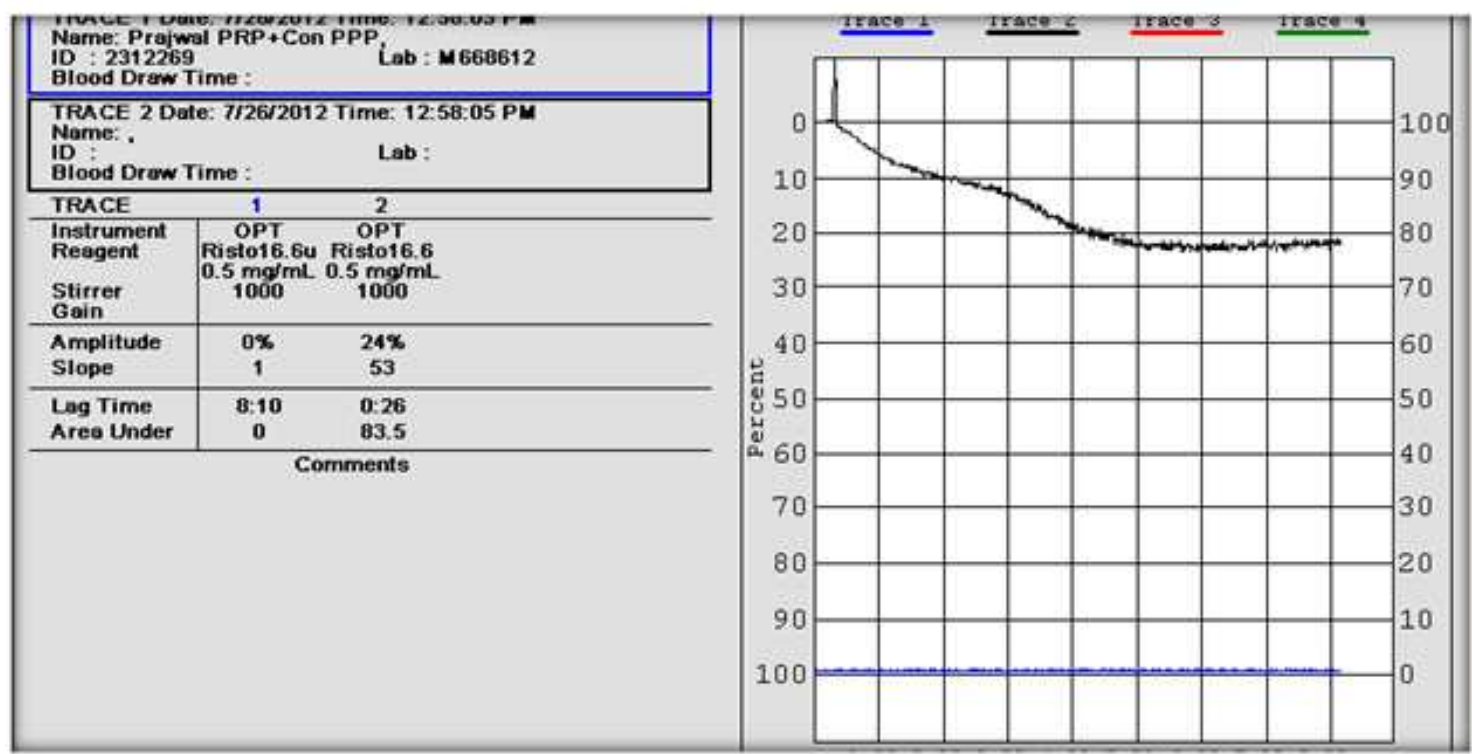

